\title{
THE CELESTIAL REFERENCE FRAME DEFINED BY VLBI
}

\author{
C. $\mathrm{Ma}$ \\ Goddard Space Flight Center \\ Geodynamics Branch 621.9 \\ Greenbelt, Maryland 20771 USA \\ and \\ D. B. Shaffer \\ Interferometrics Inc. \\ 8150 Leesburg Pike \\ Vienna, Virginia 22180 USA
}

\begin{abstract}
VLBI currently produces the most accurate positions of celestial objects. From 1979 to 1987,114 extragalactic radio sources have been observed with dual-frequency Mark III VLBI as part of the NASA Crustal Dynamics Project and the NGS POLARIS/IRIS program. The formal statistical errors of conventional celestial coordinates are as small as 0.3 milliarcseconds. The fundamental quantity measured by VLBI is the arc length between radio sources. Thus, we suggest that VLBI be used to establish a coordinate reference frame based solely on radio positions, and that this system not necessarily be coupled to right ascension and declination.
\end{abstract}

The Mark III VLBI system was developed by the Crustal Dynamics Project (CDP) of the National Aeronautics and Space Administration (NASA) to support research in geodynamics. This system is described in Clark et al. (1985). The Mark III is also used in the POLARIS and IRIS networks coordinated by the National Geodetic Survey (NGS). Since the quasi-inertial reference frame in which terrestrial motions are to be measured with centimeter precision is that defined by extragalactic radio sources, it is necessary to have correspondingly precise positions of these fiducial points.

More than 150,000 dual-frequency observations have been made of 114 radio sources to determine their positions. These observations come from more than 400 experiments, each covering approximately one day and using up to seven stations simultaneously. A large majority of the sessions are from the POLARIS/IRIS networks, but the observations are roughly equally divided between CDP and POLARIS/IRIS. About half of the sources have been used as geodetic reference sources. The others have been observed in survey sessions designed to enlarge our reference catalog. There is a wide variation in the number of observations for various sources. Some of our standard sources have been observed more than 15,000 times while some of the survey sources have been observed less than 10 times. Formal errors for the positions of the sources range from about 0.3 milliarcseconds (mas) for the most frequently observed sources, up to a few mas for sources with fewer than 50 observations.

The stations and sources are almost entirely in the northern hemisphere, and the observing baselines have predominantly east-west orientation. Recent observations to South Africa have enabled us to observe more southerly sources (down to -30 degrees declination) and to reduce the declination errors on low declination sources. 
The data were analyzed in a single, sequential least-squares solution as described in $\mathrm{Ma}$ et al. (1986). The astronomical and geophysical models generally followed the MERIT standards. However, these standards do not specify the theoretical model for the VLBI observables. In our analysis, as in all Mark I and Mark III geodetic work described and published by the VLBI groups at Goddard, NGS, and the Center for Astrophysics, the theoretical model was that of Robertson (1975). Further discussions of our VLBI radio source position measurements can be found in Ma et al. (1986) and $\mathrm{Ma}$ (1987).

The fundamental celestial quantity measured by VLBI is the arc length between radio sources, now being measured with sub-milliarcsecond accuracies. A comparison of Mark III positions with Mark II VLBI positions derived by JPL from the Deep Space Network (O. Sovers, private communication) shows a weighted mean arc length difference of 2 mas for the approximately 60 sources in common. The HIPPARCOS satellite will also measure arc length differences, of similar accuracy, for many bright stars. Neither the VLBI nor HIPPARCOS measurements require any reference to the complicated dynamics of the Earth.

Values of right ascension and declination depend on knowledge of the orientation of the earth and the definition of the origin of the right ascension system. (VLBI declinations are nominally absolute, but only with respect to the instantaneous rotation axis of the earth.) Hence, we suggest that consideration should be given to the establishment of a coordinate reference frame based solely on (relative) positions of radio sources. The relative positions of the radio sources would define a very nearly inertial frame. Positions of celestial objects would be invariant with time, except for true proper motions.

The system would be defined by the assigned positions of several sources. The relative orientations of these sources would have to be well measured and repeatable at any frequency. They should be located such that no degeneracies occur when determining the relative position of any other objects. This system could be defined independently of any consideration of earth orientation, but it should be straight forward to determine RA/Dec-like quantities for observation purposes. Thus, precession would not "move" the sources, but rather indicate where the earth is with respect to the celestial frame. The celestial frame could be aligned with some large scale structure of the universe (the ecliptic, the Galactic equator, or some super-cluster equator) for conceptual purposes, but this is not necessary.

\section{REFERENCES}

Clark, T. A. et al. (1985). IEEE Trans. Geoscience \& Remote Sensing GE-23, 438.

Ma, C. et al. (1986). Astronomical Journal 92, 1020.

$\mathrm{Ma}$, C. (1987), IAU Symposium \#128 (in press).

Robertson, D. S. (1975). Ph.D. thesis, Massachusetts Institute of Technology. 